\title{
Neural components of altruistic punishment
}

\author{
Emily Du ${ }^{1 *}$ and Steve W. C. Chang ${ }^{2,3}$ \\ ${ }^{1}$ Center for Cognitive Neuroscience, Duke Institute for Brain Sciences, Duke University, Durham, NC, USA \\ ${ }^{2}$ Department of Psychology, Yale University, New Haven, CT, USA \\ ${ }^{3}$ Department of Neurobiology, Yale University School of Medicine, New Haven, CT, USA
}

\section{Edited by:}

Hauke R. Heekeren, Freie

Universität Berlin, Germany

Reviewed by:

Sabine Windmann, Goethe

University Frankfurt, Germany

Erie Dell Boorman, University of

Oxford, UK

\section{*Correspondence:}

Emily Du, Center for Cognitive

Neuroscience, Duke Institute for

Brain Sciences, Duke University,

LSRC BIdg., Rm B353, 450

Research Drive, Durham, NC 27708,

USA

e-mail: emily.du@duke.edu
Altruistic punishment, which occurs when an individual incurs a cost to punish in response to unfairness or a norm violation, may play a role in perpetuating cooperation. The neural correlates underlying costly punishment have only recently begun to be explored. Here we review the current state of research on the neural basis of altruism from the perspectives of costly punishment, emphasizing the importance of characterizing elementary neural processes underlying a decision to punish. In particular, we emphasize three cognitive processes that contribute to the decision to altruistically punish in most scenarios: inequity aversion, cost-benefit calculation, and social reference frame to distinguish self from others. Overall, we argue for the importance of understanding the neural correlates of altruistic punishment with respect to the core computations necessary to achieve a decision to punish.

Keywords: altruistic punishment, costly punishment, inequity aversion, cost-benefit calculation, social reference frame

\section{PUNISHMENT IN A COOPERATIVE SOCIETY}

Hammurabi's Code is regarded as one of the world's oldest written legal systems, a timeless example from the ancient world of explicit, codified social norms and punishments instated against those who defected (Jarus, 2014). While most modern proceedings no longer follow lex talionis, today's societies continue to rely on cooperation between individuals to promote collective action, produce public goods, and deter free-riding (Ostrom, 2000).

Altruistic punishment occurs when an individual forgoes a personal gain to punish (Seymour et al., 2007). The biological definition of "altruism," unlike its colloquial counterpart, does not assume or impose intentionality to the actors of altruistic behavior (Wilson, 1992). Here, "altruistic" describes the sacrifice of personal gain, not the motivation of this sacrifice. In second party (SP) punishment, an individual who receives an unfair offer in a monetary exchange or suffers from a selfish investment made by a partner punishes by reducing the norm violator's payout at a cost (Fehr and Gächter, 2002; Egas and Riedl, 2008), or rejects the unfair offer such that both players have a reduced payout (Güth and Tietz, 1990). Third party (TP) punishment, on the other hand, occurs when an uninvolved individual punishes the violator at a cost (Fehr and Fischbacher, 2004; Bernhard et al., 2006).

Altruistic punishment generates a vast array of questions about its behavioral and neural mechanisms. The evolutionary consequences for promoting prosocial behaviors, such as cooperation, remain continuously debated (Nakamaru and Iwasa, 2006; Rand et al., 2010; Rand and Nowak, 2013; Peysakhovich et al., 2014) but lie outside the scope of this paper; we instead focus on neural mechanisms that could be at play when deciding to costly punish. Our knowledge could benefit from a framework that incorporates neural computations contributing to altruistic punishment. Here, we explore the current literature on neuroscientific studies of operationally-defined altruistic punishment, and emphasize three cognitive processes that guide the decision to altruistically punish, namely inequity aversion, cost-benefit calculation, and social reference frame used for distinguishing self from others. These three cognitive processes have distinct neural correlates, as evidenced by literature that will be discussed, which could relate to the neural mechanism that underlies the complex decision of altruistic punishment. Therefore, connecting the literature on altruistic punishment to that of these three cognitive processes could inform our understanding of the neural correlates of punishment.

\section{NEURAL CORRELATES OF ALTRUISTIC PUNISHMENT}

How does the brain mediate altruistic punishment? Many studies have directly explored this question (Table 1). Blood-oxygen-level dependent (BOLD) signals in the bilateral anterior insula (AI), dorsolateral prefrontal cortex (DLPFC), and anterior cingulate cortex (ACC) are all associated with receiving unfair vs. fair offers from another individual (Sanfey et al., 2003). Overall, converging evidence seems to suggest an involvement of reinforcement via the striatum in mediating altruistic punishment. One study compared BOLD activity of participants punishing as the SP and TP during a dictator game, and found differential activations in the right nucleus accumbens (NAc) and DLPFC (Strobel et al., 2011). Another study found greater activations in DLPFC and caudate nucleus $(\mathrm{CdN})$ among other regions when individuals received unfair offers (Spitzer et al., 2007). Greater CdN activation has also been associated with costly compared to symbolic punishment (i.e., no reduction in endowment for either player), in which individuals' willingness to incur a greater cost to punish was associated with stronger CdN activations (de Quervain et al., 2004; White et al., 2014). Intriguingly, reducing serotonin signaling during the ultimatum game (UG) can lead to an increase in the 


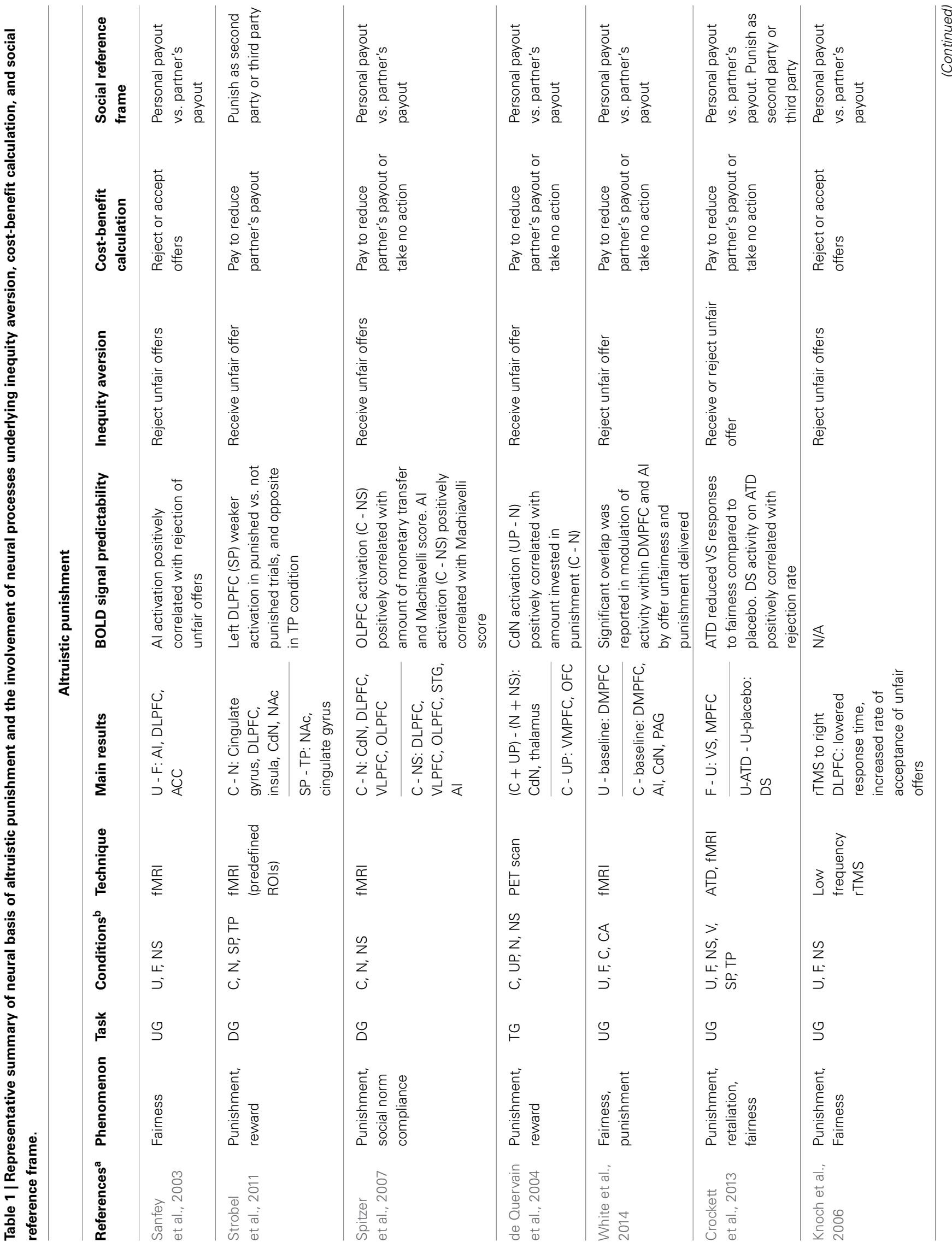




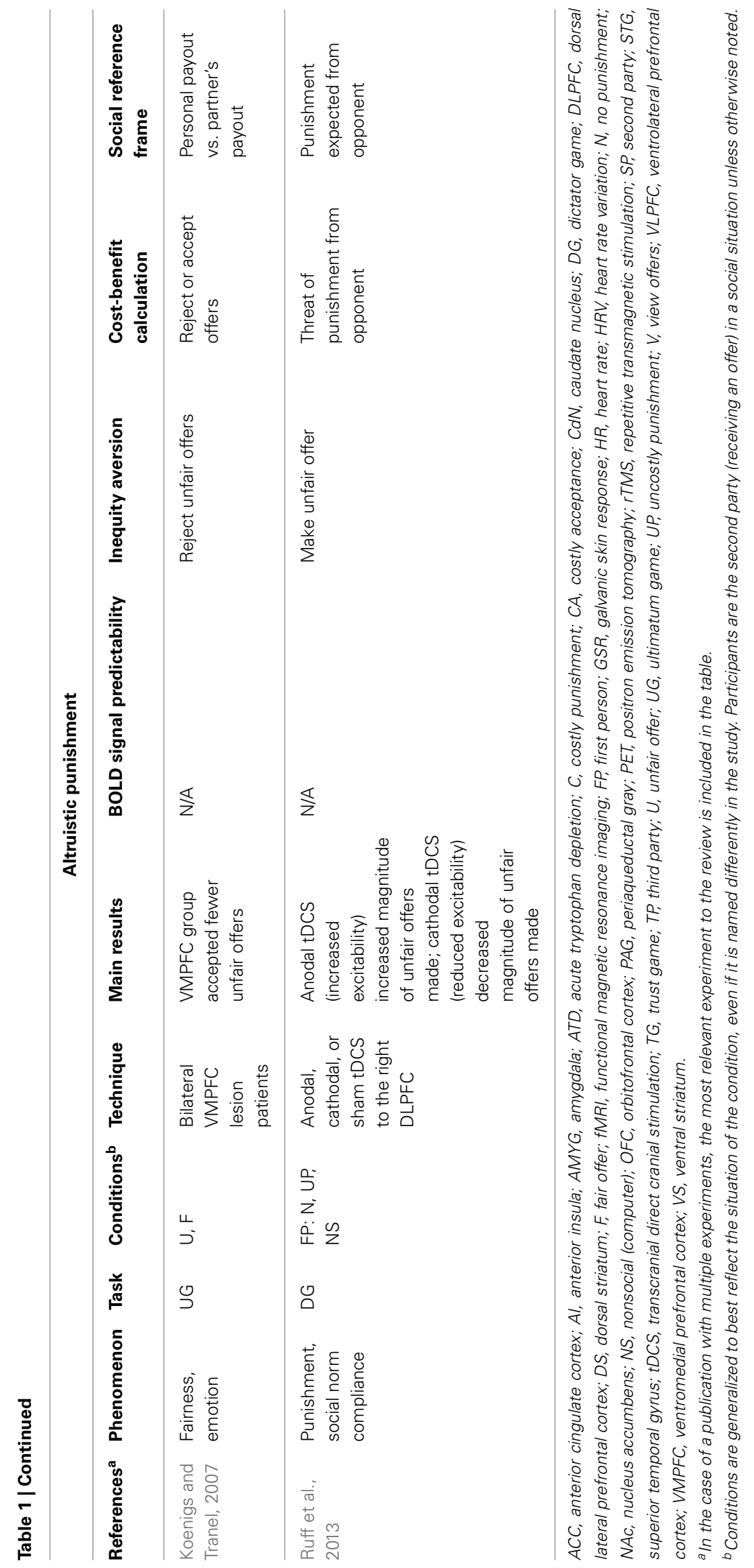


likelihood of punishing others who responded to them unfairly by modulating striatal activations (Crockett et al., 2013), suggesting that serotonin may set the sensitivity threshold for fairnessand punishment-related processing.

Furthermore, converging evidence suggests that prefrontal regions are specialized for evaluating fairness and guiding norm compliance during punishment. In addition to the previously cited work implicating the DLFPC in administering punishment as the SP, low-frequency repetitive transmagnetic stimulation to the right DLPFC increased the acceptance rate of unfair offers in the UG (Knoch et al., 2006). By contrast, participants with bilateral lesions in the ventromedial prefrontal cortex (VMPFC) exhibited the opposite pattern, accepting fewer unfair offers (Koenigs and Tranel, 2007). Furthermore, increasing or decreasing neuronal excitability using transcranial direct current stimulation in the right lateral prefrontal cortex (rLPFC) differentially controls sanction-free compared to sanction-induced transfers, without affecting the conceptualization of fairness norm and sanctions, when a player makes a monetary offer in either a dictator or UG context (Ruff et al., 2013). These results demonstrate that rLPFC is causally involved in norm compliance, but in strikingly different ways depending on the presence of punishment threats.

However, it remains unclear what computations are primarily driving the neural signals in altruistic punishment tasks. In the following sections, we highlight three fundamental cognitive processes occurring in most altruistic punishment scenarios that might play significant roles in driving the neural activations. These processes may inform how different neural circuits are implemented in driving altruistic punishment.

\section{INEQUITY AVERSION}

Altruistic punishment occurs in response to an offer perceived to be inequitable. Inequity often evokes negative affect, which may motivate the decision to altruistically punish (Montague and Lohrenz, 2007). Using a resource-sharing task, Haruno and Frith (2010) showed that BOLD signals from amygdala (AMYG) predict individual differences in aversion to inequity, such that prosocial orientation is driven by an intuitive aversion for the inequitable division of resources across self and others. Furthermore, in a task asking participants to allocate goods between two groups of children, high insula activity was associated with choosing an equitable allocation vs. an inequitable one, supporting the role of the insula for signaling inequity associated with a norm violation (Hsu et al., 2008) (Figure 1A). This finding suggests that individuals with stronger negative affective signaling from the bilateral AI reduce inequity due to a greater sensitivity to the violation of fairness (Hsu et al., 2008). Unreciprocated cooperation, which could be conceptualized as a violation of one's expectation based on the social norm, also increases activation in the bilateral AI, as well as the left AMYG (Rilling et al., 2008). AMYG activity during a decision-making task with losses and gains correlated with loss aversion (Sokol-Hessner et al., 2013). Taken together, social exchanges with an inequitable outcome seem to recruit neural systems, including the AI and AMYG, involved in affective signaling to influence norm compliance by modulating fairness perception.
Because inequity motivates a punishing action, neural processes associated with inequity detection and aversion could significantly guide the decision to costly punish. The aversion associated with inequity, on the other hand, may reflect how the neural signals corresponding to inequity detection are transformed and further processed by emotion-related circuitry. Converging evidence indeed indicates that task events correlated with inequity typically activate brain regions implicated in affective processing. Further research is needed to understand how these signals influence the decision to punish.

In realistic settings, there are not necessarily tight temporal relations between the time of inequity detection and the punishment-some punishments may occur as an immediate reaction, whereas others may occur long after the infraction, many days and even years following inequity detection. In the laboratory, most studies have had focused on relatively small time window between inequity detection and punishment for practical purposes. The role of inequity-evoked negative affect is likely to be greater when the time from the inequity detection to the punishing act is relatively short. It would be interesting to test the magnitude of affective drive in different brain regions during punishing decisions as a function of the delay between the time of inequity detection and forming the decision to punish.

\section{COST-BENEFIT CALCULATION}

Cost-benefit analysis could occur when deciding to carry out altruistic punishment, requiring that the cost of punishment be weighed against the benefit of punishing (Egas and Riedl, 2008). The costly punishment decision could hinge upon the value representation of the possible outcomes (Sugrue et al., 2005), which subsequently may depend on the severity of the opponent's infraction, the ratio of monetary cost to punish, the impact of the punishment itself on the opponent, as well as any expected future gains from punishing.

In examining effort as a proxy for cost required for some benefit, a lesion to ACC reduced a rat's willingness to expend effort to receive a large reward, whereas control animals typically expended energy (Walton et al., 2003). When human subjects performed a similar effort-based task, BOLD activity in the dorsal ACC and striatum increased with respect to the net value of the outcome rather than the amount of effort anticipated (Croxson et al., 2009). Single-unit recordings in rat ACC during a choice task of two options differing in cost-benefit ratio found a population of neurons with elevated firing rates during the pursuit of a high cost, high reward option, an effect that did not solely reflect variations in physical effort or food acquisition, suggesting the ACC's role in integrating information about cost and reward (Hillman and Bilkey, 2010) (Figure 1B). Furthermore, in a competitive setting, a population of ACC neurons signals the choice with the greatest net utility based on the cost of competing and the benefit of reward (Hillman and Bilkey, 2012). Inactivating ACC in mice decreases their preference for the option requiring greater effort (Hosking et al., 2014). Although such efforts related to acquiring a reward or completing a task may not be directly translatable to the monetary loss in costly punishment, they share the basic principle of incurring a cost to potentially obtain a desired outcome. 
A

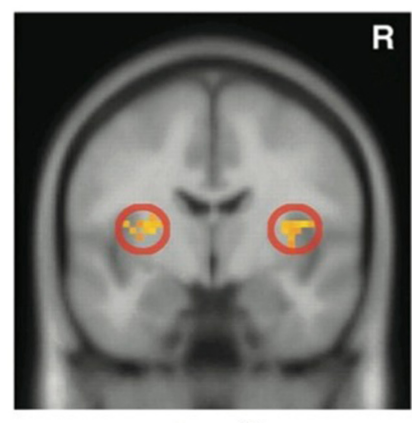

$y=-5$

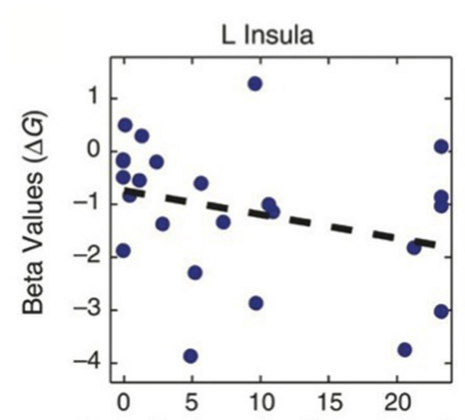

Inequity Aversion Parameter $\left(\alpha_{i}\right)$

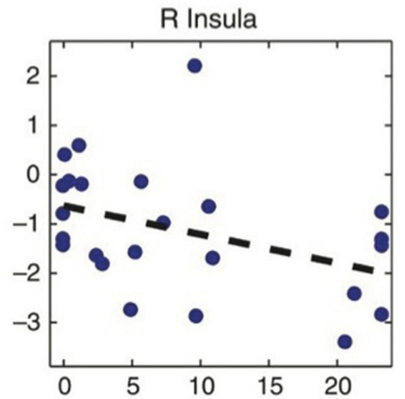

iii.
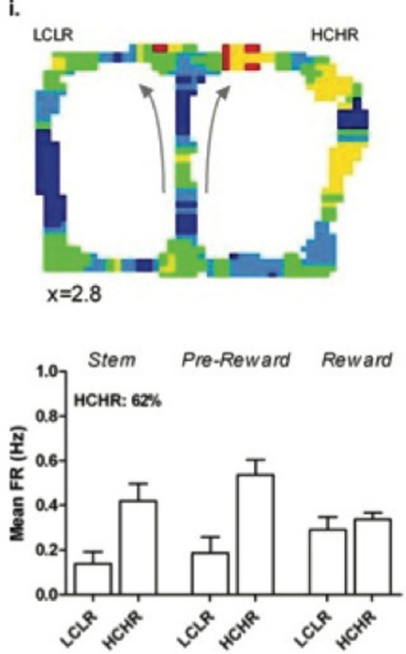

ii.

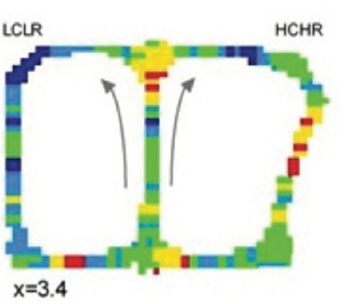

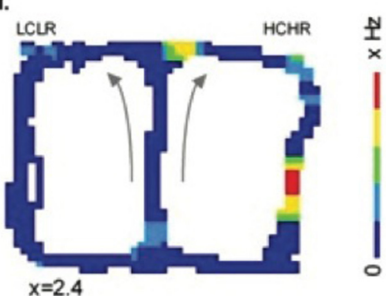
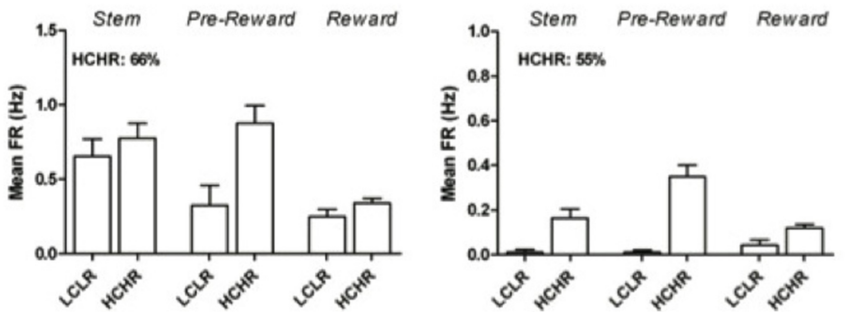
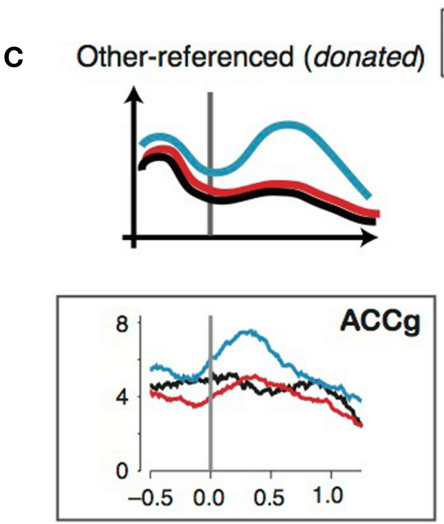

- Rewards delivered to oneself Rewards delivered to another
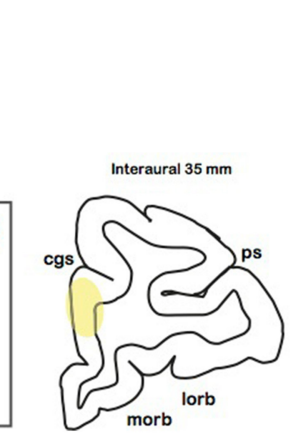

FIGURE 1 | Representative studies on the neural components of altruistic punishment. (A) Left: Hsu et al. (2008) found that activation in the bilateral insula (left) is negatively correlated with inequity in a task involving allocating differential benefits across two groups of children. Right: Individual's activations (beta values) were negatively correlated with individual inequity-aversion parameters. Modified from Hsu et al. (2008) with permission. (B) In the study by Hillman and Bilkey (2010), mice navigated through a maze in which they either chose a "low cost, low reward" (LCLR) arm (left) or a "high cost, high reward" (HCHR) arm (right). For each of the three typical HCHR-biased ACC cells shown here (i-iii), firing rates that are pseudo-color mapped onto the spatial position
Commonly-referenced, both-referenced
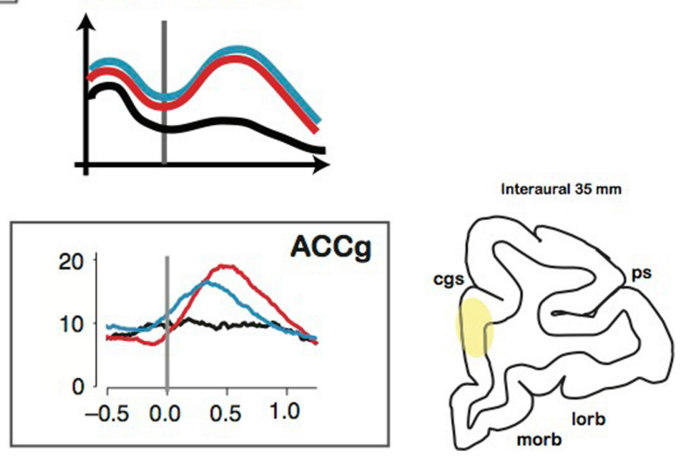

of the maze illustrate overall higher activity for the HCHR compare to the LCLR option. The bar graphs show the mean firing rates (FR) across different spatial positions ("epochs") for HCHR and LCLR choices for each cell. The percentage of $\mathrm{HCHR}$ choices is also indicated. Modified from Hillman and Bilkey (2010) with permission. (C) Left:

Other-referenced representation of rewards allocated to another monkey in the room found in the subpopulation of neurons in the gyrus of the anterior cingulate cortex (ACCg). Right: Mirrored (commonly-referenced) representation of rewards received by an actor and another monkey found in the subpopulation of ACCg neurons. Modified from Chang et al. (2013) and Chang (2013), with permission. 
Some studies have related a nonsocial reward currency with social rewards, which could be occurring in altruistic punishment when a player sacrifices money to punish another player. An exchange rate between viewing social images and receiving monetary rewards that falls along a distribution correlated with the valuation of the image is reflected in the hemodynamic activity of the posterior VMPFC (Smith et al., 2010). Functional connectivity of the VMPFC to temporal-parietal junction, MPFC, middle temporal gyrus, and posterior cingulate cortex suggests a network governing subjective valuations of social reward (Smith et al., 2014). Weighing the cost and benefit in the social reward domain is not a behavior restricted to humans. Rhesus macaques also are willing to exchange small amounts of juice rewards in order to view socially salient images but require juice overpayment to view less desirable images (Deaner et al., 2005). Importantly, the specific amount of juice sacrificed or demanded falls along a distribution that is correlated with the valuation of the image, generating an exchange rate between social (image) and nonsocial (juice) rewards. Similar to such an exchange rate between monetary (or primary reinforcer in monkeys) and social reward, participants forgo a monetary reward to punish in SP or TP punishment, in which the loss is compensated by the reduction in their opponent's endowment. If punishing the defector is in fact rewarding (de Quervain et al., 2004), the punishing cost could be thought in part as being exchanged with a social reward.

Regardless of whether the gains of altruistic punishment are monetary or social, cost-benefit calculations may play a key role in the neural processes leading up to a decision to punish at a predicted cost. Examining cost-benefit relations in both nonsocial rewards and social rewards and associated neural activity across different individuals during costly punishment decisions may reveal intriguing insights into the mechanisms underlying the decision to costly punish.

\section{SOCIAL REFERENCE FRAME}

Any behavior that involves another individual requires a set of representations across self and others. Costly punishment, in particular, may require the evaluation of others' internal states when an individual is faced with outcomes that could ultimately benefit another individual or a group. Evidence demonstrates that there are shared networks in the brain that compute information with respect to self and others (Decety and Sommerville, 2003). Importantly, these shared representations seem to be generalized to multiple cognitive domains. The brain regions related to the affective response to pain, including the rostral portion of ACC, bilateral insula (both medial and anterior), and the brainstem, are activated in groups who either receive an electric shock to their own finger or watch a significant other receive a shock (Singer et al., 2004). Furthermore, it was shown that observing another person experiencing pain activates a broad range of brain areas across frontal and parietal cortices as well as AMYG, areas that are implicated in emotional and social cue processing (Ochsner et al., 2008). Likewise, a TP observer could take on a SP perspective such that another player's misfortune is construed as his own. A recent study suggests that some brain areas may process selfspecific misfortune but other areas may project other's misfortune onto one's own. Corradi-Dell'Acqua et al. (2013) found selective activation in MPFC when participants were shown unfair offers involving themselves but not others, whereas AI activations were also associated with unfair offers to others (2013). Whether "perspective-taking" or "affective projection" is a prerequisite to promoting TP remains to be explored.

In addition, the TP observer likely infers the reward contingencies from the perspectives of the two players after punishing. Such multidimensional inferences across oneself and another individual must require a signal transformation across self and others (Chang, 2013) (Figure 1C). Insight into this transformation could be obtained by examining how reward outcomes across self and others are encoded in the brain. A recent study tested such encoding mechanisms when pairs of rhesus macaques were engaged in a social reward exchange paradigm (Chang et al., 2013). When an actor monkey was choosing to deliver juice rewards between himself and a recipient (Self/Other) as well as between the recipient and no one (Other/Neither), anatomically distinct regions of the primate frontal cortex encoded the reward outcomes across self and others using different social reference frames. Neurons in OFC primarily signaled the received rewards of the actor monkey, whereas neurons in the sulcus of ACC (ACCs) predominantly signaled the foregone rewards of the actor. Notably, one subpopulation of neurons in ACC gyrus (ACCg) exclusively signaled the rewards received by the recipient, whereas another subpopulation mirrored the rewards received by the actor or the recipient. In addition to these "other-regarding" neurons, another subpopulation in ACCg exclusively encoded one's own reward outcomes. The specialized function of ACCg in signaling the rewarding events of others was also recently reported in a human neuroimaging study (Apps and Ramnani, 2014). An accurate readout of self- and other-referenced reward information could be critical for mediating the concept of actor and recipient during altruistic punishment.

In both SP and TP punishment, the punisher must be able to accurately process affective responses and reward outcomes as a result of punishment across himself and the other player or players involved. As such information is essential to behaviors directed at other individuals, it is critical to comprehend the amount and specific nature of neural activations driven by such computations in social punishment scenarios.

\section{CONCLUDING REMARKS}

Costly punishment poses some of the most interesting evolutionary questions for scientists. Cooperation, which rests inherently on the shared needs of individuals and groups, seems to be at complete odds with traditional evolutionary theories that pit individuals against each other in a race for survival and reproduction. Yet cooperation in animal and human behavior often determines the survival of entire populations. The neural underpinnings of altruistic punishment in humans are actively being investigated, but many questions remain unanswered. Several studies so far suggest the lateral aspect of the prefrontal cortex as a key locus in mediating altruistic punishment (Knoch et al., 2006; Spitzer et al., 2007; Strobel et al., 2011; Ruff et al., 2013). These regions may reflect the computations of cognitive variables. However, some studies also highlight the importance of the insula and AMYG for their role in affective processing during 
such punishments (Hsu et al., 2008; Haruno and Frith, 2010). The neural processes related to these cognitive and affective variables are likely to interact with one another up until the time of punishment. Such ongoing interactions may provide the basis for internal deliberation on a decision to punish, as well as mediating a potential change of mind before the execution of that decision.

A paradigm with high temporal sensitivity may reveal how cognitive and affective signals in the brain converge or diverge across the entire timespan of deciding to punish at a cost. For example, future investigations combining fMRI with another method allowing for a higher temporal resolution, such as electroencephalography or functional near-infrared spectroscopy, may reveal new, crucial information on the region-to-region interactions between neural signals correlated with inequity aversion, cost-benefit calculation, and information processing across self and others. Furthermore, developing a nonhuman primate model of costly punishment may complement research in humans by providing more detailed neuronal mechanisms of the three core neural processes involved in altruistic punishment through single-unit recording and pharmacological interventions of specific populations of neurons. Another important factor to consider in any social neuroscience research is the context in which a given social behavior takes place. Understanding how social context gates the neural processes associated with inequity aversion, cost-benefit calculation, and information processing across self and others will better inform the complex contingencies behind altruistic punishment.

\section{ACKNOWLEDGMENTS}

We thank David G. Rand for his helpful comments on the manuscript. This work was supported by a Duke Undergraduate Research Support Office grant (Emily Du) and R00-MH099093 (Steve W. C. Chang).

\section{REFERENCES}

Apps, M. A. J., and Ramnani, N. (2014). The anterior cingulate gyrus signals the net value of others' rewards. J. Neurosci. 34, 6190-6200. doi: 10.1523/JNEUROSCI.2701-13.2014

Bernhard, H., Fischbacher, U., and Fehr, E. (2006). Parochial altruism in humans. Nature 442, 912-915. doi: 10.1038/nature04981

Chang, S. W. C. (2013). Coordinate transformation approach to social interactions. Front. Neurosci. 7:147. doi: 10.3389/fnins.2013.00147

Chang, S. W. C., Gariépy, J.-F., and Platt, M. L. (2013). Neuronal reference frames for social decisions in primate frontal cortex. Nat. Neurosci. 16, 243-250. doi: 10.1038/nn.3287

Corradi-Dell'Acqua, C., Civai, C., Rumiati, R. I., and Fink, G. R. (2013). Disentangling self-and fairness-related neural mechanisms involved in the ultimatum game: an fMRI study. Soc. Cogn. Affect. Neurosci. 8, 424-431. doi: 10.1093/scan/nss014

Crockett, M. J., Apergis-Schoute, A., Herrmann, B., Lieberman, M. D., Müller, U., Robbins, T. W., et al. (2013). Serotonin modulates striatal responses to fairness and retaliation in humans. J. Neurosci. 33, 3505-3513. doi: 10.1523/JNEUROSCI.2761-12.2013

Croxson, P. L., Walton, M. E., O’Reilly, J. X., Behrens, T. E. J., and Rushworth, M. F. S. (2009). Effort-based cost-benefit valuation and the human brain. J. Neurosci. 29, 4531-4541. doi: 10.1523/JNEUROSCI.4515-08.2009

Deaner, R. O., Khera, A. V., and Platt, M. L. (2005). Monkeys pay per view: adaptive valuation of social images by rhesus macaques. Curr. Biol. 15, 543-548. doi: 10.1016/j.cub.2005.01.044

Decety, J., and Sommerville, J. A. (2003). Shared representations between self and other: a social cognitive neuroscience view. Trends Cogn. Sci. 7, 527-533. doi: 10.1016/j.tics.2003.10.004 de Quervain, D. J. F., Fischbacher, U., Treyer, V., Schellhammer, M., Schnyder, U., Buck, A., et al. (2004). The neural basis of altruistic punishment. Science 305, 1254-1258. doi: 10.1126/science. 1100735

Egas, M., and Riedl, A. (2008). The economics of altruistic punishment and the maintenance of cooperation. Proc. R. Soc. B Biol. Sci. 275, 871-878. doi: 10.1098/rspb.2007.1558

Fehr, E., and Fischbacher, U. (2004). Third-party punishment and social norms. Evol. Hum. Behav. 25, 63-87. doi: 10.1016/S1090-5138(04) 00005-4

Fehr, E., and Gächter, S. (2002). Altruistic punishment in humans. Nature 415, 137-140. doi: 10.1038/415137a

Güth, W., and Tietz, R. (1990). Ultimatum bargaining behavior: a survey and comparison of experimental results. J. Econ. Psychol. 11, 417-449. doi: 10.1016/01674870(90)90021-Z

Haruno, M., and Frith, C. D. (2010). Activity in the amygdala elicited by unfair divisions predicts social value orientation. Nat. Neurosci. 13, 160-161. doi: 10.1038/nn.2468

Hillman, K. L., and Bilkey, D. K. (2010). Neurons in the rat anterior cingulate cortex dynamically encode cost-benefit in a spatial decision-making task. J. Neurosci. 30, 7705-7713. doi: 10.1523/JNEUROSCI.1273-10.2010

Hillman, K. L., and Bilkey, D. K. (2012). Neural encoding of competitive effort in the anterior cingulate cortex. Nat. Neurosci. 15, 1290-1297. doi: 10.1038/nn.3187

Hosking, J. G., Cocker, P. J., and Winstanley, C. A. (2014). Dissociable contributions of anterior cingulate cortex and basolateral amygdala on a rodent cost/benefit decision-making task of cognitive effort. Neuropsychopharmacology 39, 1558-1567. doi: 10.1038/npp.2014.27

Hsu, M., Anen, C., and Quartz, S. R. (2008). The right and the good: distributive justice and neural encoding of equity and efficiency. Science 320, 1092-1095. doi: 10.1126/science.1153651

Jarus, O. (2014). Code of Hammurabi: Ancient Babylonian Laws 2013 [cited March 13, 2014]. Available online at: http://www.livescience.com/39393-codeof-hammurabi.html

Knoch, D., Pascual-Leone, A., Meyer, K., Treyer, V., and Fehr, E. (2006). Diminishing reciprocal fairness by disrupting the right prefrontal cortex. Science 314, 829-832. doi: 10.1126/science. 1129156

Koenigs, M., and Tranel, D. (2007). Irrational economic decision-making after ventromedial prefrontal damage: evidence from the ultimatum game. J. Neurosci. 27, 951-956. doi: 10.1523/JNEUROSCI.4606-06.2007

Montague, P. R., and Lohrenz., T. (2007). To detect and correct: norm violations and their enforcement. Neuron 56, 14-18. doi: 10.1016/j.neuron.2007. 09.020

Nakamaru, M., and Iwasa, Y. (2006). The coevolution of altruism and punishment: role of the selfish punisher. J. Theor. Biol. 240, 475-488. doi: 10.1016/j.jtbi.2005.10.011

Ochsner, K. N., Zaki, J., Hanelin, J., Ludlow, D. H., Knierim, K., Ramachandran, T., et al. (2008). Your pain or mine? Common and distinct neural systems supporting the perception of pain in self and other. Soc. Cogn. Affect. Neurosci. 3, 144-160. doi: 10.1093/scan/nsn006

Ostrom, E. (2000). Collective action and the evolution of social norms. J. Econ. Perspect. 14, 137-158. doi: 10.1257/jep.14.3.137

Peysakhovich, A., Nowak, M. A., and Rand D. G. (2014). Humans display a 'cooperative phenotype' that is domain general and temporally stable. Nat. Commun. 5. doi: 10.1038/ncomms5939

Rand, D. G., Armao, J. J. IV., Nakamaru, M., and Ohtsuki, H. (2010). Anti-social punishment can prevent the co-evolution of punishment and cooperation. J. Theor. Biol. 265, 624-632. doi: 10.1016/j.jtbi.2010.06.010

Rand, D. G., and Nowak, M. A. (2013). Human cooperation. Trends Cogn. Sci. 17, 413. doi: 10.1016/j.tics.2013.06.003

Rilling, J. K., Goldsmith, D. R., Glenn, A. L., Jairam, M. R., Elfenbein, H. A., Dagenais, J. E., et al. (2008). The neural correlates of the affective response to unreciprocated cooperation. Neuropsychologia 46, 1256-1266. doi: 10.1016/j.neuropsychologia.2007.11.033

Ruff, C. C., Ugazio, G., and Fehr, E. (2013). Changing social norm compliance with noninvasive brain stimulation. Science 342, 482-484. doi: 10.1126/science. 1241399

Sanfey, A. G., Rilling, J. K., Aronson, J. A., Nystrom, L. E., and Cohen, J. D. (2003). The neural basis of economic decision-making in the ultimatum game. Science 300, 1755-1758. doi: 10.1126/science.1082976 
Seymour, B., Singer, T., and Dolan, R. (2007). The neurobiology of punishment. Nat. Rev. Neurosci. 8, 300-311. doi: 10.1038/nrn2119

Singer, T., Seymour, B., O’Doherty, J., Kaube, H., Dolan, R. J., and Frith, C. D. (2004). Empathy for pain involves the affective but not sensory components of pain. Science 303, 1157-1162. doi: 10.1126/science.1093535

Smith, D. V., Clithero, J. A., Boltuck, S. E., and Huettel, S. A. (2014). Functional connectivity with ventromedial prefrontal cortex reflects subjective value for social rewards. Soc. Cogn. Affect. Neurosci. 9, 2017-2025. doi: 10.1093/scan/nsu005

Smith, D. V., Hayden, B. Y., Truong, T.-K., Song, A. W., Platt, M. L., and Huettel, S. A. (2010). Distinct value signals in anterior and posterior ventromedial prefrontal cortex. J. Neurosci. 30, 2490-2495. doi: 10.1523/JNEUROSCI.331909.2010

Sokol-Hessner, P., Camerer, C. F., and Phelps, E. A. (2013). Emotion regulation reduces loss aversion and decreases amygdala responses to losses. Soc. Cogn. Affect. Neurosci 8, 341-350. doi: 10.1093/scan/nss002

Spitzer, M., Fischbacher, U., Herrnberger, B., Grön, G., and Fehr, E. (2007). The neural signature of social norm compliance. Neuron 56, 185-196. doi: 10.1016/j.neuron.2007.09.011

Strobel, A., Zimmermann, J., Schmitz, A., Reuter, M., Lis, S., Windmann, S., et al. (2011). Beyond revenge: neural and genetic bases of altruistic punishment. Neuroimage 54, 671-680. doi: 10.1016/j.neuroimage.2010. 07.051

Sugrue, L. P., Corrado, G. S., and Newsome, W. T. (2005). Choosing the greater of two goods: neural currencies for valuation and decision making. Nat. Rev. Neurosci. 6, 363-375. doi: 10.1038/nrn1666
Walton, M. E., Bannerman, D. M., Alterescu, K., and Rushworth, M. F. S. (2003). Functional specialization within medial frontal cortex of the anterior cingulate for evaluating effort-related decisions. J. Neurosci. 23, 6475-6479.

White, S. F., Brislin, S. J., Sinclair, S., and Blair, J. R. (2014). Punishing unfairness: rewarding or the organization of a reactively aggressive response? Hum. Brain Mapp. 35, 2137-2147. doi: 10.1002/hbm.22316

Wilson, D. S. (1992). On the relationship between evolutionary and psychological definitions of altruism and selfishness. Biol. Philos. 7, 61-68. doi: 10.1007/BF00130164

Conflict of Interest Statement: The authors declare that the research was conducted in the absence of any commercial or financial relationships that could be construed as a potential conflict of interest.

Received: 09 October 2014; accepted: 16 January 2015; published online: 09 February 2015.

Citation: Du E and Chang SWC (2015) Neural components of altruistic punishment. Front. Neurosci. 9:26. doi: 10.3389/fnins.2015.00026

This article was submitted to Decision Neuroscience, a section of the journal Frontiers in Neuroscience.

Copyright (c) 2015 Du and Chang. This is an open-access article distributed under the terms of the Creative Commons Attribution License (CC BY). The use, distribution or reproduction in other forums is permitted, provided the original author(s) or licensor are credited and that the original publication in this journal is cited, in accordance with accepted academic practice. No use, distribution or reproduction is permitted which does not comply with these terms. 